Journal of Advanced Computer Science \& Technology, 5(2) (2017) 20-32
Journal of Advanced Computer Science \& Technology
Website: www.sciencepubco.com/index.php/JACST
doi: $10.14419 / j a c s t . v 6 i 2.7011$
Research paper

\title{
A heuristic to predict the optimal pattern-growth direction for the pattern growth-based sequential pattern mining approach
}

\author{
Kenmogne Edith Belise ${ }^{1 *}$, Nkambou Roger ${ }^{2}$, Tadmon Calvin $^{3}$ and Engelbert MEPHU NGUIFO ${ }^{4}$ \\ ${ }^{1,3}$ Faculty of Science, Department of Mathematics and Computer Science, LIFA, Po. Box. 67 Dschang, Cameroon, \\ ${ }^{2}$ Computer Science Department, University of Québec at Montréal, 201 avenue du président-Kennedy Montréal (Québec) H2X $3 Y 7$ Canada, \\ Knowledge Management laboratory, \\ ${ }^{4}$ Computer Science Institute - LIMOS - UMR CNRS 6158 Complexe scientifique des cézeaux, 1 rue de la chebarde, 63178 Aubière cedex 49, \\ Bd François-Mitterrand - CS 6003263001 Clermont-Ferrand Cedex 1 \\ *Corresponding author E-mail:ebkenmogne@gmail.com
}

\begin{abstract}
Sequential pattern mining is an efficient technique for discovering recurring structures or patterns from very large datasets, with a very large field of applications. It aims at extracting a set of attributes, shared across time among a large number of objects in a given database. Previous studies have developed two major classes of sequential pattern mining methods, namely, the candidate generation-and-test approach based on either vertical or horizontal data formats represented respectively by GSP and SPADE, and the pattern-growth approach represented by FreeSpan, PrefixSpan and their further extensions. The performances of these algorithms depend on how patterns grow. Because of this, we introduce a heuristic to predict the optimal pattern-growth direction, i.e. the pattern-growth direction leading to the best performance in terms of runtime and memory usage. Then, we perform a number of experimentations on both real-life and synthetic datasets to test the heuristic. The performance analysis of these experimentations show that the heuristic prediction is reliable in general.
\end{abstract}

Keywords: sequence mining; sequential pattern; frequent pattern; pattern-growth direction; heuristic; prefixspan; suffixspan

\section{Introduction}

Many real world applications have to deal with sequential data. Discovery of sequential patterns $[13,5]$ from large dataset was first introduced by Agrawal and Srikant [3] in 1995 based on their study of customer purchase sequences, as follows: Given a set of sequences, where each sequence consists of a list of events (or elements) and each event consists of a set of items, and given a user-specified minimum support threshold of min_sup, sequential pattern mining finds all frequent subsequences, that is, the subsequences whose occurrence frequency in the set of sequences is no less than min_sup. Sequential pattern mining is one of the important fields in data mining because of its variety of applications in web access pattern analysis, market basket analysis, fault detection in network, DNA sequences etc. It plays a vital role in different areas. It is essentially an enumeration problem over the sub-sequence partial order looking for those sequences that are frequent. The search can be performed in a breadth-first or depth-first manner, starting with more general (shorter) sequences and extending them towards more specific (longer) ones. Many algorithms were proposed for sequential pattern mining. The existing algorithms essentially differ in the data structures used to index the database to facilitate fast enumeration. These mining algorithms are broadly classified into two approaches known as the Apriori-based candidate generation approach $[3,4,8,9,10,14,18,3,20,22,23]$ and the pattern growth approach $[11,17,15,16,12,19]$.
Since the first proposal of this data mining task and its associated efficient mining algorithms, there has been a growing number of researchers in the field and tremendous progress [13] has been made, evidenced by hundreds of follow-up research publications, on various kinds of extensions and applications, ranging from scalable data mining methodologies, to handling a wide diversity of data types, various extended mining tasks, and a variety of new applications.

Improvements in sequential pattern mining algorithms have followed similar trend in the related area of association rule mining and have been motivated by the need to process more data at a faster speed with lower cost. Previous studies have developed two major classes of sequential pattern mining methods : The Apriori-based approach $[3,4,8,9,10,14,18,3,20,22,23]$ and the pattern growth approach $[11,17,15,16,12,19]$.

The Apriori-based approach form the vast majority of algorithms proposed in the literature for sequential pattern mining. Apriori-like algorithms depend mainly on the Apriori anti-monotony property, which states the fact that any super-pattern of an infrequent pattern cannot be frequent, and are based on a candidate generation-and-test paradigm proposed in association rule mining $[1,2]$. This candidate generation-and-test paradigm is carried out by GSP [3], SPADE [23], and SPAM [4]. Mining algorithms derived from this approach are based on either vertical or horizontal data formats. Algorithms based on the vertical data format involve AprioriAll, AprioriSome and DynamicSome [3], GSP [3], PSP [14] and SPIRIT [8], while those based on the horizontal data format involve SPADE [23], cSPADE 
[22], SPAM [4], LAPIN-SPAM [20], IBM [18] and PRISM [9, 10]. The generation-and-test paradigm has the disadvantage of repeatedly generating an explosive number of candidate sequences and scanning the database to maintain the support count information for these sequences during each iteration of the algorithm, which makes them computationally expensive. To increase the performance of these algorithms constraint driven discovery can be carried out. With constraint driven approach system should concentrate only on user specific or user interested patterns or user specified constraints such as minimum support, minimum gap or time interval etc. With regular expression these constraints are studied in SPIRIT [8].

To alleviate these problems, the pattern-growth approach, represented by FreeSpan [11], PrefixSpan $[15,16]$ and their further extensions, namely FS-Miner [6], LAPIN [12, 21], SLPMiner [19] and WAP-mine [17], for efficient sequential pattern mining adopts a divide-and-conquer pattern growth paradigm as follows. Sequence databases are recursively projected into a set of smaller projected databases based on the current sequential patterns, and sequential patterns are grown in each projected database by exploring only locally frequent fragments $[11,16]$. The frequent pattern growth paradigm removes the need for the candidate generation and prune steps that occur in the Apriori-based algorithms and repeatedly narrows the search space by dividing a sequence database into a set of smaller projected databases, which are mined separately. The major advantage of projection-based sequential pattern-growth algorithms is that they avoid the candidate generation and prune steps that occur in the Apriori-based algorithms. They grow longer sequential patterns from the shorter frequent ones. The major cost of these algorithms is the cost of forming projected databases recursively. To alleviate this problem, a pseudo-projection method is exploited to reduce this cost. Instead of performing physical projection, one can register the index (or identifier) of the corresponding sequence and the starting position of the projected suffix in the sequence. Then, a physical projection of a sequence is replaced by registering a sequence identifier and the projected position index point. Pseudo-projection reduces the cost of projection substantially when the projected database can fit in main memory.

PrefixSpan [15, 16] and FreeSpan [11] differ at the criteria of partitionning projected databases and at the criteria of growing patterns. FreeSpan creates projected databases based on the current set of frequent patterns without a particular ordering (i.e., pattern-growth direction), whereas PrefixSpan projects databases by growing frequent prefixes. Thus, PrefixSpan follows the unidirectional growth whereas FreeSpan follows the bidirectional growth. Another difference between FreeSpan and PrefixSpan is that the pseudo-projection works efficiently for PrefixSpan but not so for FreeSpan. This is because for PrefixSpan, an offset position clearly identifies the suffix and thus the projected subsequence. However, for FreeSpan, since the next step pattern-growth can be in both forward and backward directions from any position, one needs to register more information on the possible extension positions in order to identify the remainder of the projected subsequences. Note that the mining can proceed from the suffix, which is essentially SuffixSpan $[15,16]$, an algorithm symmetric to PrefixSpan by growing suffixes from the end of the sequence forward. The main difference between PrefixSpan and SuffixSpan is that PrefixSpan follows the left-to-right pattern-growth direction while SuffixSpan follows the right-to-left pattern-growth direction. PrefixSpan $[15,16]$ performs much better than GSP[3], SPADE [23] and FreeSpan[11]. The major cost of PrefixSpan is the construction of projected databases.

This paper studies the problem of determining the optimal patterngrowth direction between left-to-right and right-to-left patterngrowth directions, i.e the pattern-growth direction leading to the best performance in terms of runtime and memory usage. To this end, a heuristic is introduced to predict the optimal pattern-growth direction. Then, a number of experimentations on both real-life and synthetic datasets are performed to test the heuristic. The performance analysis of these experimentations show that the heuristic prediction is reliable in general.

The rest of the paper is organized as follows. Section 2 states the problem of mining sequential patterns. Section 3 proposes a heuristic to predict the optimal pattern-growth direction. Section 4 presents experimental results and analyses them. Sub-section 4.1 presents real-life and synthetic datasets used in experimentations. Sub-section 4.2 presents experimental results. Sub-section 4.3 is devoted to performance analysis. Concluding remarks are given in section 5 .

\section{Statement of the problem of mining sequen- tial patterns}

The problem of mining sequential patterns, and its associated notation, can be given as follows:

Let $I=\left\{i_{1}, i_{2}, \ldots, i_{n}\right\}$ be a set of literals, termed items, which comprise the alphabet. An itemset is a subset of items. A sequence is an ordered list of itemsets. A sequence $s$ is denoted by $\prec s_{1}, s_{2}, \ldots s_{n} \succ$, where $s_{j}$ is an itemset. $s_{j}$ is also called an element of the sequence, and denoted as $\left(x_{1}, x_{2}, \ldots, x_{m}\right)$, where $x_{k}$ is an item. For brevity, the brackets are omitted if an element has only one item, i.e. element $(x)$ is written as $x$. An item can occur at most once in an element of a sequence, but can occur multiple times in different elements of a sequence. The number of instances of items in a sequence is called the length of the sequence. A sequence with length $l$ is called an l-sequence. The length of a sequence $\alpha$ is denoted $|\alpha|$ A sequence $\alpha=\prec a_{1} a_{2} \ldots a_{n} \succ$ is called subsequence of another sequence $\beta=\prec b_{1} b_{2} \ldots b_{m} \succ$ and $\beta$ a supersequence of $\alpha$, denoted as $\alpha \subseteq \beta$, if there exist integers $1 \leq j_{1}<j_{2}<\ldots<j_{n} \leq j_{m}$ such that $a_{1} \subseteq b_{j 1}, a_{2} \subseteq b_{j 2}, \ldots, a_{n} \subseteq b_{j n}$. Symbol $\varepsilon$ denotes the empty sequence.

We are given a database $S$ of input-sequences. A sequence database is a set of tuples of the form $\prec s i d, s \succ$ where sid is a sequence id and $s$ a sequence. A tuple $\prec$ sid, $s \succ$ is said to contain a sequence $\alpha$, if $\alpha$ is a subsequence of $s$. The support of a sequence $\alpha$ in a sequence database $S$ is the number of tuples in the database containing $\alpha$, i.e.

$$
\operatorname{support}(S, \alpha)=|\{\prec \operatorname{sid}, s \succ \mid \prec \operatorname{sid}, s \succ \in S \wedge \alpha \subseteq s\}| .
$$

It can be denoted as support $(\alpha)$ if the sequence database is clear from the context. Given a user-specified positive integer denoted min_support, termed the minimum support or the support threshold, a sequence $\alpha$ is called a sequential pattern in the sequence database $S$ if $\operatorname{support}(S, \alpha) \geq$ min_support. A sequential pattern with length $l$ is called an l-pattern. Given a sequence database and the min_support threshold, sequential pattern mining is to find the complete set of sequential patterns in the database.

\section{Right-to-left direction versus left-to-right di- rection}

In this section, we introduce a heuristic which recommends a patterngrowth direction between left-to-right direction (also called PrefixSpan) and right-to-left direction (also called SuffixSpan).

Let consider a dataset $S$, an item $x_{p}$, an itemset $s_{j}=\left(x_{1}, x_{2}, \ldots, x_{m}\right)$, a sequence $s=\prec s_{1}, s_{2}, \ldots s_{n} \succ$ and a support threshold st. The weight Weight $\left(x_{p}, S, s t\right)$ of item $x_{p}$ in dataset $S$ according to threshold st is defined as.

Weight $\left(x_{p}, S, s t\right)=\left\{\begin{array}{rlr}\operatorname{Support}\left(S, x_{p}\right) \text { if } & \operatorname{Support}\left(S, x_{p}\right) \geq s t \\ 0 \text { if } & \operatorname{Support}\left(S, x_{p}\right)<s t\end{array}\right.$

The weight Weight $\left(s_{j}, S, s t\right)$ of itemset $s_{j}$ in dataset $S$ according to threshold $s t$ is defined as follows.

Weight $\left(s_{j}, S, s t\right)=\sum_{p=1}^{m} \operatorname{Weight}\left(x_{p}, S, s t\right)$, 
The left weight LeftWeight $(S, s)$ of sequence $s$ in dataset $S$ according to threshold $s t$ is defined as

$\operatorname{LeftWeight}(s, S, s t)=\sum_{j=1}^{s i z e}(\operatorname{size}-j+1) \operatorname{Weight}\left(s_{j}, S, s t\right)$,

where size $=n / 2$. Similarly, the right weight $\operatorname{RightWeight}(S, s)$ of sequence $s$ in dataset $S$ according to threshold $s t$ is defined as

$\operatorname{RightWeight}(s, S, s t)=\sum_{j=l e f t I n d e x}^{n}(j-$ leftIndex +1$) W e i g h t\left(s_{j}, S, s t\right)$,

where leftIndex $=n-$ size +1 . The cumulative left weight LeftWeight $(S, s t)$ of all the sequences in dataset $S$ according to threshold $s t$ is defined as

LeftWeight $(S, s t)=\sum_{s \in S} \operatorname{LeftWeight}(s, S, s t)$.

Similarly, the cumulative right weight $\operatorname{RightWeight}(S)$ of all the sequences in dataset $S$ according to threshold $s t$ is defined by

$\operatorname{RightWeight}(S, s t)=\sum_{s \in S} \operatorname{RightWeight}(s, S, s t)$.

Recommendations are performed as follows. The left-to-right direction (also called PrefixSpan) is recommended if LeftWeight $(S)<$ RightWeight $(S)$. The right-to-left direction (also called SuffixSpan) is recommended if RightWeight $(S)<\operatorname{LeftWeight}(S)$.

\section{Experimental results and performance ana- lysis}

\subsection{Presentation of datasets}

The datasets used here are collected from the webpage (http://www.philippe-fournier-viger.com/spmf/index.php) of SPMF software [7]. This webpage provides large datasets in SPMF format that are often used in the data mining litterature for evaluating and comparing algorithm performance.

\subsubsection{Real-life datasets}

BMSWebView1 (Gazelle) ( KDD CUP 2000) : This dataset contains 59,601 sequences of clickstream data from an e-commerce. It contains 497 distinct items. It is called here BMS1_spmf. The average length of sequences is 2.42 items with a standard deviation of 3.22. In this dataset, there are some long sequences. For example, 318 sequences contains more than 20 items.

BMSWebView2 (Gazelle) ( KDD CUP 2000) : This is a second dataset (called here BMS2) used in the KDD-CUP 2000 competition. It contains 77,512 sequences of clickstream data. It contains 3340 distinct items. The average length of sequences is 4.62 items with a standard deviation of 6.07 items.

Kosarak : This is a very large dataset containing 990000 sequences of clickstream data from an hungarian news portal. The dataset in its original format can be found at http://fimi.ua.ac.be/data/. The SPMF format is called Kosarak_converted. However, this dataset is very large. Therefore, a subset of only 10000 sequences (kosarak10k) and a subset of 25000 sequences (kosarak25k) are provided in the SPMF format.

SIGN : This is a dataset of sign language utterance containing approximately 800 sequences. The original dataset file in another format can be obtained here with more details on this dataset.
BIBLE : This dataset is a conversion of the Bible into a sequence database (each word is an item). It contains 36369 sequences and 13905 distinct items. The average length of a sequence is 21.6 items. The average number of distinct items per sequence is 17.84 .

LEVIATHAN : This dataset is a conversion of the novel Leviathan by Thomas Hobbes (1651) as a sequence database (each word is an item). It contains 5834 sequences and 9025 distinct items. The average number of items per sequence is : 33.8. The average number of distinct items per sequence is 26.34 .

MSNBC : This is a dataset of clickstream data. The original dataset contains 989,818 sequences obtained from the UCI repository. Here the shortest sequences have been removed to keep only 31,790 sequences. The number of distinct items in this dataset is 17 (an item is a webpage category). The average number of itemsets per sequence is 13.33 . The average number of distinct items per sequence is 5.33 .

FIFA : A dataset of 20,450 sequences of clickstream data from the website of FIFA World Cup 98. It has 2,990 distinct items. The average sequence length is 34.74 items with a standard deviation of 24.08 items. This dataset was created by processing a part of the web logs from the World Cup 98.

\subsubsection{Synthetic datasets}

The synthetic datasets used here are collected from the webpage of SPMF software [7]. To generate synthetic sequence databases, you can use the sequence database generator provided in SPMF, which is flexible and easy to use. It is also possible to generate sequence databases by using the IBM Generator. Files generated by the IBM Generator can be converted in SPMF format by using the conversion tool provided in SPMF. Another alternative for generating synthetic sequences databases is to use a Matlab program provided by Ashwin Balani. Here are some synthetic sequence databases generated with the IBM Quest Dataset Generator for Sequential Pattern Mining, converted to the SPMF format:

1: data.slen_10.tlen_1.seq.patlen_2.lit.patlen_8.nitems_5000_spmf

2: data.slen_10.tlen_1.seq.patlen_3.lit.patlen_8.nitems_5000_spmf

3: data.slen_8.tlen_1.seq.patlen_5.lit.patlen_8.nitems_5000_spmf

4: data.slen_8.tlen_1.seq.patlen_6.lit.patlen_8.nitems_5000_spmf

\subsection{Experimental results}

We exhaustively experimented on both real world and synthetic datasets presented in section 4.1 in order to assess the performance of our approach.

All experiments are done on a 4-cores of $2.16 \mathrm{GHz}$ Intel(R) Pentium(R) CPU N3530 with 4 gigabytes main memory, running Ubuntu 14.04 LTS. All the algorithms are implemented in Java and grounded on SPMF software [7].

The experiments consisted of running the pattern-growth algorithms related to the left-to-right and right-to-left directions on each dataset while decreasing the support threshold until algorithms became too long to execute or ran out of memory. For each dataset, we recorded the number of frequent patterns discorved, the execution time, the memory usage and the pattern-growth direction recommeded by the heuristic. The experimental results are presented in tables. The execution times of the heuristic (without the time of loading data) are not presented as they are negligible compared to the execution times of the two mining algorithms, i.e PrefixSpan and SuffixSpan. 
Table 1: Execution times (in milliseconds) and memory sizes (in mb) performances following the two pattern-growth directions and heuristic prediction on the real-life dataset BIBLE.

\begin{tabular}{|c|c|c|c|c|c|c|}
\hline \multicolumn{2}{|c|}{ Dataset BIBLE } & \multicolumn{2}{|c|}{ Left-to-right } & \multicolumn{2}{|c|}{ Right-to-left } & \multirow{2}{*}{$\begin{array}{l}\text { Heuristic } \\
\text { Prediction }\end{array}$} \\
\hline $\begin{array}{l}\text { Threshold } \\
\text { (in \%) }\end{array}$ & $\begin{array}{l}\text { Pattern } \\
\text { count }\end{array}$ & $\begin{array}{c}\text { Runtime } \\
\text { (in ms) }\end{array}$ & $\begin{array}{l}\text { Memory } \\
\text { (in mb) }\end{array}$ & $\begin{array}{l}\text { Runtime } \\
\text { (in ms) }\end{array}$ & $\begin{array}{l}\text { Memory } \\
\text { (in mb) }\end{array}$ & \\
\hline 0.02 & 5293 & 553637 & 930.267 & 455317 & 876.033 & Right-to-left \\
\hline 0.03 & 2216 & 226704 & 721.167 & 181669 & 673.497 & Right-to-left \\
\hline 0.04 & 1185 & 117672 & 595.961 & 92805 & 572.001 & Right-to-left \\
\hline 0.05 & 774 & 73706 & 544.005 & 57235 & 517.643 & Right-to-left \\
\hline 0.06 & 507 & 51357 & 517.617 & 39998 & 486.966 & Right-to-left \\
\hline 0.07 & 371 & 36581 & 501.661 & 29194 & 454.309 & Right-to-left \\
\hline
\end{tabular}

Table 2: Execution times (in milliseconds) and memory sizes (in mb) performances following the two pattern-growth directions and heuristic prediction on the real-life dataset LEVIATHAN.

\begin{tabular}{|c|c|c|c|c|c|c|}
\hline \multicolumn{2}{|c|}{ Dataset LEVIATHAN } & \multicolumn{2}{|c|}{ Left-to-right } & \multicolumn{2}{|c|}{ Right-to-left } & \multirow{2}{*}{$\begin{array}{l}\text { Heuristic } \\
\text { Prediction }\end{array}$} \\
\hline $\begin{array}{l}\text { Threshold } \\
\text { (in \%) }\end{array}$ & $\begin{array}{l}\text { Pattern } \\
\text { count }\end{array}$ & $\begin{array}{l}\text { Runtime } \\
\text { (in ms) }\end{array}$ & $\begin{array}{l}\text { Memory } \\
\text { (in mb) }\end{array}$ & $\begin{array}{l}\text { Runtime } \\
\text { (in ms) }\end{array}$ & $\begin{array}{l}\text { Memory } \\
\text { (in mb) }\end{array}$ & \\
\hline 0.02 & 33387 & 378601 & 682.674 & 484164 & 767.991 & Left-to-right \\
\hline 0.03 & 12534 & 199897 & 531.461 & 271939 & 568.760 & Left-to-right \\
\hline 0.04 & 6286 & 128508 & 490.904 & 178264 & 516.615 & Left-to-right \\
\hline 0.05 & 3698 & 91745 & 477.662 & 126534 & 472.160 & Left-to-right \\
\hline 0.06 & 2300 & 63960 & 441.430 & 87292 & 435.925 & Left-to-right \\
\hline 0.07 & 1577 & 49631 & 426.322 & 69908 & 409.915 & Left-to-right \\
\hline
\end{tabular}

Table 3: Execution times (in milliseconds) and memory sizes (in mb) performances following the two pattern-growth directions and heuristic prediction on the real-life dataset MSNBC.

\begin{tabular}{|c|c|c|c|c|c|c|}
\hline \multicolumn{2}{|c|}{ Dataset MSNBC } & \multicolumn{2}{|c|}{ Left-to-right } & \multicolumn{2}{|c|}{ Right-to-left } & \multirow{2}{*}{$\begin{array}{l}\text { Heuristic } \\
\text { Prediction }\end{array}$} \\
\hline $\begin{array}{l}\text { Threshold } \\
\text { (in \%) }\end{array}$ & $\begin{array}{l}\text { Pattern } \\
\text { count }\end{array}$ & $\begin{array}{l}\text { Runtime } \\
\text { (in ms) }\end{array}$ & $\begin{array}{l}\text { Memory } \\
\text { (in mb) }\end{array}$ & $\begin{array}{l}\text { Runtime } \\
\text { (in ms) }\end{array}$ & $\begin{array}{l}\text { Memory } \\
\text { (in mb) }\end{array}$ & \\
\hline 0.38 & 13 & 10677 & 535.886 & 10538 & 467.362 & Right-to-left \\
\hline 0.39 & 13 & 10699 & 500.293 & 10531 & 499.377 & Right-to-left \\
\hline 0.40 & 13 & 10712 & 518.660 & 10363 & 496.498 & Right-to-left \\
\hline 0.41 & 12 & 10493 & 509.333 & 10136 & 498.207 & Right-to-left \\
\hline 0.42 & 11 & 10067 & 511.344 & 10004 & 464.402 & Right-to-left \\
\hline 0.43 & 11 & 10099 & 475.897 & 9897 & 508.419 & Right-to-left \\
\hline
\end{tabular}


Table 4: Execution times (in milliseconds) and memory sizes (in mb) performances following the two pattern-growth directions and heuristic prediction on the real-life dataset FIFA.

\begin{tabular}{|c|c|c|c|c|c|c|}
\hline \multicolumn{2}{|c|}{ Dataset FIFA } & \multicolumn{2}{|c|}{ Left-to-right } & \multicolumn{2}{|c|}{ Right-to-left } & \multirow{2}{*}{$\frac{\text { Heuristic }}{\text { Prediction }}$} \\
\hline $\begin{array}{l}\text { Threshold } \\
\text { (in \%) }\end{array}$ & $\begin{array}{l}\text { Pattern } \\
\text { count }\end{array}$ & $\begin{array}{l}\text { Runtime } \\
\text { (in ms) }\end{array}$ & $\begin{array}{l}\text { Memory } \\
\text { (in mb) }\end{array}$ & $\begin{array}{l}\text { Runtime } \\
\text { (in ms) }\end{array}$ & $\begin{array}{l}\text { Memory } \\
\text { (in mb) }\end{array}$ & \\
\hline 0.20 & 934 & 41786 & 557.274 & 35459 & 539.499 & Right-to-left \\
\hline 0.21 & 737 & 35441 & 511.141 & 29795 & 518.640 & Right-to-left \\
\hline 0.22 & 557 & 27387 & 469.111 & 25036 & 486.738 & Right-to-left \\
\hline 0.23 & 403 & 21638 & 443.496 & 20000 & 440.658 & Right-to-left \\
\hline 0.24 & 318 & 18019 & 430.906 & 16885 & 424.836 & Right-to-left \\
\hline 0.25 & 277 & 16290 & 419.911 & 14934 & 396.822 & Right-to-left \\
\hline
\end{tabular}

Table 5: Execution times (in milliseconds) and memory sizes (in mb) performances following the two pattern-growth directions and heuristic prediction on the real-life dataset BMS1_spmf.

\begin{tabular}{|c|c|c|c|c|c|c|}
\hline \multicolumn{2}{|c|}{ Dataset BMS1_spmf } & \multicolumn{2}{|c|}{ Left-to-right } & \multicolumn{2}{|c|}{ Right-to-left } & \multirow{2}{*}{$\begin{array}{l}\text { Heuristic } \\
\text { Prediction }\end{array}$} \\
\hline $\begin{array}{l}\text { Threshold } \\
\text { (in \%) }\end{array}$ & $\begin{array}{l}\text { Pattern } \\
\text { count }\end{array}$ & $\begin{array}{l}\text { Runtime } \\
\text { (in ms) }\end{array}$ & $\begin{array}{l}\text { Memory } \\
\text { (in mb) }\end{array}$ & $\begin{array}{l}\text { Runtime } \\
\text { (in ms) }\end{array}$ & $\begin{array}{l}\text { Memory } \\
\text { (in mb) }\end{array}$ & \\
\hline 0.01 & 77 & 544 & 101.077 & 528 & 101.188 & Right-to-left \\
\hline 0.02 & 22 & 232 & 53.360 & 230 & 54.084 & Right-to-left \\
\hline 0.03 & 11 & 169 & 54.025 & 169 & 53.438 & Right-to-left \\
\hline 0.04 & 5 & 128 & 45.337 & 128 & 45.347 & Left-to-right \\
\hline 0.05 & 4 & 122 & 44.098 & 121 & 44.522 & Left-to-right \\
\hline 0.06 & 3 & 112 & 43.452 & 116 & 43.443 & Left-to-right \\
\hline
\end{tabular}

Table 6: Execution times (in milliseconds) and memory sizes (in mb) performances following the two pattern-growth directions and heuristic prediction on the real-life dataset BMS1_spmf.

\begin{tabular}{|c|c|c|c|c|c|c|}
\hline \multicolumn{2}{|c|}{ Dataset BMS1_spmf } & \multicolumn{2}{|c|}{ Left-to-right } & \multicolumn{2}{|c|}{ Right-to-left } & \multirow{2}{*}{$\begin{array}{l}\text { Heuristic } \\
\text { Prediction }\end{array}$} \\
\hline $\begin{array}{l}\text { Threshold } \\
\text { (in \%) }\end{array}$ & $\begin{array}{l}\text { Pattern } \\
\text { count }\end{array}$ & $\begin{array}{c}\text { Runtime } \\
\text { (in ms) }\end{array}$ & $\begin{array}{l}\text { Memory } \\
\text { (in mb) }\end{array}$ & $\begin{array}{c}\text { Runtime } \\
\text { (in ms) }\end{array}$ & $\begin{array}{c}\text { Memory } \\
\text { (in mb) }\end{array}$ & \\
\hline 0.0010 & 3991 & 6803 & 217.695 & 6506 & 222.413 & Right-to-left \\
\hline 0.0011 & 3010 & 5942 & 206.596 & 5536 & 196.607 & Right-to-left \\
\hline 0.0012 & 2382 & 5320 & 194.733 & 4974 & 183.731 & Right-to-left \\
\hline 0.0013 & 1961 & 4935 & 183.512 & 4611 & 184.140 & Right-to-left \\
\hline 0.0014 & 1686 & 4596 & 178.610 & 4350 & 173.401 & Right-to-left \\
\hline 0.0015 & 1428 & 4312 & 171.280 & 4056 & 170.134 & Right-to-left \\
\hline
\end{tabular}


Table 7: Execution times (in milliseconds) and memory sizes (in mb) performances following the two pattern-growth directions and heuristic prediction on the real-life dataset BMS2.

\begin{tabular}{|c|c|c|c|c|c|c|}
\hline \multicolumn{2}{|c|}{ Dataset BMS2 } & \multicolumn{2}{|c|}{ Left-to-right } & \multicolumn{2}{|c|}{ Right-to-left } & \multirow{2}{*}{$\begin{array}{l}\text { Heuristic } \\
\text { Prediction }\end{array}$} \\
\hline $\begin{array}{l}\text { Threshold } \\
\text { (in \%) }\end{array}$ & $\begin{array}{l}\text { Pattern } \\
\text { count }\end{array}$ & $\begin{array}{c}\text { Runtime } \\
\text { (in ms) }\end{array}$ & $\begin{array}{c}\text { Memory } \\
\text { (in mb) }\end{array}$ & $\begin{array}{c}\text { Runtime } \\
\text { (in ms) }\end{array}$ & $\begin{array}{l}\text { Memory } \\
\text { (in mb) }\end{array}$ & \\
\hline 0.0010 & 23294 & 31424 & 460.304 & 15792 & 353.775 & Right-to-left \\
\hline 0.0011 & 17820 & 27545 & 439.496 & 14414 & 359.994 & Right-to-left \\
\hline 0.0012 & 14029 & 24160 & 407.116 & 13166 & 342.035 & Right-to-left \\
\hline 0.0013 & 11594 & 21890 & 376.972 & 12195 & 337.262 & Right-to-left \\
\hline 0.0014 & 9401 & 19168 & 359.311 & 11235 & 339.543 & Right-to-left \\
\hline 0.0015 & 7840 & 17361 & 355.599 & 10471 & 330.676 & Right-to-left \\
\hline
\end{tabular}

Table 8: Execution times (in milliseconds) and memory sizes (in mb) performances following the two pattern-growth directions and heuristic prediction on the real-life dataset SIGN.

\begin{tabular}{|c|c|c|c|c|c|c|}
\hline \multicolumn{2}{|c|}{ Dataset SIGN } & \multicolumn{2}{|c|}{ Left-to-right } & \multicolumn{2}{|c|}{ Right-to-left } & \multirow{2}{*}{$\frac{\text { Heuristic }}{\text { Prediction }}$} \\
\hline $\begin{array}{c}\text { Threshold } \\
\text { (in \%) }\end{array}$ & $\begin{array}{l}\text { Pattern } \\
\text { count }\end{array}$ & $\begin{array}{c}\text { Runtime } \\
\text { (in ms) }\end{array}$ & $\begin{array}{l}\text { Memory } \\
\text { (in mb) }\end{array}$ & $\begin{array}{l}\text { Runtime } \\
\text { (in ms) }\end{array}$ & $\begin{array}{l}\text { Memory } \\
\text { (in mb) }\end{array}$ & \\
\hline 0.10 & 105544 & 54587 & 809.646 & 52698 & 791.261 & Right-to-left \\
\hline 0.11 & 78002 & 44124 & 660.625 & 43204 & 677.467 & Right-to-left \\
\hline 0.12 & 59065 & 35821 & 577.677 & 36039 & 606.811 & Right-to-left \\
\hline 0.13 & 45460 & 30486 & 523.951 & 30928 & 519.777 & Right-to-left \\
\hline 0.14 & 34365 & 24706 & 438.836 & 25558 & 464.903 & Right-to-left \\
\hline 0.15 & 27213 & 20686 & 435.898 & 21978 & 330.676 & Right-to-left \\
\hline
\end{tabular}

Table 9: Execution times (in milliseconds) and memory sizes (in mb) performances following the two pattern-growth directions and heuristic prediction on the real-life dataset kosarak10k.

\begin{tabular}{|c|c|c|c|c|c|c|}
\hline \multicolumn{2}{|c|}{ Dataset kosarak10k } & \multicolumn{2}{|c|}{ Left-to-right } & \multicolumn{2}{|c|}{ Right-to-left } & \multirow{2}{*}{$\begin{array}{c}\text { Heuristic } \\
\text { Prediction }\end{array}$} \\
\hline $\begin{array}{l}\text { Threshold } \\
\text { (in \%) }\end{array}$ & $\begin{array}{c}\text { Pattern } \\
\text { count }\end{array}$ & $\begin{array}{l}\text { Runtime } \\
\text { (in ms) }\end{array}$ & $\begin{array}{c}\text { Memory } \\
\text { (in mb) }\end{array}$ & $\begin{array}{l}\text { Runtime } \\
\text { (in ms) }\end{array}$ & $\begin{array}{c}\text { Memory } \\
\text { (in mb) }\end{array}$ & \\
\hline 0.0015 & 88111 & 28667 & 407.745 & 9526 & 354.966 & Right-to-left \\
\hline 0.0017 & 31060 & 11560 & 352.353 & 5444 & 341.654 & Right-to-left \\
\hline 0.0019 & 19477 & 8006 & 346.310 & 4012 & 335.946 & Right-to-left \\
\hline 0.0020 & 16655 & 7285 & 346.767 & 3634 & 294.289 & Right-to-left \\
\hline 0.0022 & 14670 & 6669 & 345.432 & 3403 & 291.439 & Right-to-left \\
\hline 0.0023 & 11265 & 5504 & 340.230 & 2942 & 285.448 & Right-to-left \\
\hline
\end{tabular}


Table 10: Execution times (in milliseconds) and memory sizes (in mb) performances following the two pattern-growth directions and heuristic prediction on the real-life dataset kosarak25k.

\begin{tabular}{|c|c|c|c|c|c|c|}
\hline \multicolumn{2}{|c|}{ Dataset kosarak25k } & \multicolumn{2}{|c|}{ Left-to-right } & \multicolumn{2}{|c|}{ Right-to-left } & \multirow{2}{*}{$\begin{array}{c}\text { Heuristic } \\
\text { Prediction }\end{array}$} \\
\hline $\begin{array}{l}\text { Threshold } \\
\text { (in \%) }\end{array}$ & $\begin{array}{l}\text { Pattern } \\
\text { count }\end{array}$ & $\begin{array}{l}\text { Runtime } \\
\text { (in ms) }\end{array}$ & $\begin{array}{c}\text { Memory } \\
\text { (in mb) }\end{array}$ & $\begin{array}{c}\text { Runtime } \\
\text { (in ms) }\end{array}$ & $\begin{array}{c}\text { Memory } \\
\text { (in mb) }\end{array}$ & \\
\hline 0.0015 & 72778 & 65839 & 553.194 & 14700 & 406.531 & Right-to-left \\
\hline 0.0020 & 14705 & 16660 & 331.601 & 7399 & 278.002 & Right-to-left \\
\hline 0.0025 & 8396 & 11077 & 298.574 & 5131 & 238.042 & Right-to-left \\
\hline 0.0030 & 4957 & 7711 & 242.761 & 4010 & 210.039 & Right-to-left \\
\hline 0.0035 & 6049 & 6049 & 227.242 & 3297 & 184.912 & Right-to-left \\
\hline 0.0040 & 2645 & 4924 & 195.322 & 2868 & 171.507 & Right-to-left \\
\hline
\end{tabular}

Table 11: Execution times (in milliseconds) and memory sizes (in mb) performances following the two pattern-growth directions and heuristic prediction on the real-life dataset Kosarak_converted.

\begin{tabular}{|c|c|c|c|c|c|c|}
\hline \multicolumn{2}{|c|}{ Dataset Kosarak } & \multicolumn{2}{|c|}{ Left-to-right } & \multicolumn{2}{|c|}{ Right-to-left } & \multirow{2}{*}{$\begin{array}{l}\text { Heuristic } \\
\text { Prediction }\end{array}$} \\
\hline $\begin{array}{l}\text { Threshold } \\
\text { (in \%) }\end{array}$ & $\begin{array}{l}\text { Pattern } \\
\text { count }\end{array}$ & $\begin{array}{c}\text { Runtime } \\
\text { (in ms) }\end{array}$ & $\begin{array}{l}\text { Memory } \\
\text { (in mb) }\end{array}$ & $\begin{array}{c}\text { Runtime } \\
\text { (in ms) }\end{array}$ & $\begin{array}{l}\text { Memory } \\
\text { (in mb) }\end{array}$ & \\
\hline 0.00160 & 92129 & 275767 & 933.843 & & & Right-to-left \\
\hline 0.00163 & 69537 & 208065 & 932.711 & 36847 & 766.786 & Right-to-left \\
\hline 0.00165 & 62940 & 186873 & 931.178 & 35762 & 749.994 & Right-to-left \\
\hline 0.00170 & 47761 & 143341 & 837.263 & 32545 & 695.050 & Right-to-left \\
\hline 0.00180 & 27250 & 86661 & 683.175 & 27271 & 544.967 & Right-to-left \\
\hline 0.00190 & 18255 & 61624 & 610.035 & 23422 & 510.269 & Right-to-left \\
\hline
\end{tabular}

Table 12: Execution times (in milliseconds) and memory sizes (in mb) performances following the two pattern-growth directions and heuristic prediction on the synthetic dataset data.slen_10.tlen_1.seq.patlen_3.1it.patlen_8.nitems_5000_spmf.

\begin{tabular}{|c|c|c|c|c|c|c|}
\hline \multicolumn{2}{|c|}{ Synthetic dataset } & \multicolumn{2}{|c|}{ Left-to-right } & \multicolumn{2}{|c|}{ Right-to-left } & \multirow{2}{*}{$\begin{array}{c}\text { Heuristic } \\
\text { Prediction }\end{array}$} \\
\hline $\begin{array}{l}\text { Threshold } \\
\text { (in \%) }\end{array}$ & $\begin{array}{c}\text { Pattern } \\
\text { count }\end{array}$ & $\begin{array}{c}\text { Runtime } \\
\text { (in ms) }\end{array}$ & $\begin{array}{r}\text { Memory } \\
\text { (in mb) }\end{array}$ & $\begin{array}{c}\text { Runtime } \\
\text { (in ms) }\end{array}$ & $\begin{array}{r}\text { Memory } \\
\text { (in mb) }\end{array}$ & \\
\hline 0.0010 & 172897 & 16127 & 778.533 & 18038 & 780.393 & Right-to-left \\
\hline 0.0011 & 119236 & 11498 & 609.879 & 11444 & 608.268 & Right-to-left \\
\hline 0.0012 & 93881 & 9300 & 527.715 & 9292 & 527.741 & Right-to-left \\
\hline 0.0013 & 62149 & 7698 & 413.225 & 7733 & 411.667 & Right-to-left \\
\hline 0.0014 & 26596 & 3678 & 290.741 & 3648 & 280.505 & Right-to-left \\
\hline 0.0015 & 23706 & 3229 & 261.430 & 3249 & 257.898 & Right-to-left \\
\hline
\end{tabular}


Table 13: Execution times (in milliseconds) and memory sizes (in mb) performances following the two pattern-growth directions and heuristic prediction on the synthetic dataset data.slen_10.tlen_1.seq.patlen_2.lit.patlen_8.nitems_5000_spmf.

\begin{tabular}{|c|c|c|c|c|c|c|}
\hline \multicolumn{2}{|c|}{ Synthetic dataset } & \multicolumn{2}{|c|}{ Left-to-right } & \multicolumn{2}{|c|}{ Right-to-left } & \multirow{2}{*}{$\begin{array}{l}\text { Heuristic } \\
\text { Prediction }\end{array}$} \\
\hline $\begin{array}{l}\text { Threshold } \\
\text { (in \%) }\end{array}$ & $\begin{array}{l}\text { Pattern } \\
\text { count }\end{array}$ & $\begin{array}{l}\text { Runtime } \\
\text { (in ms) }\end{array}$ & $\begin{array}{l}\text { Memory } \\
\text { (in mb) }\end{array}$ & $\begin{array}{c}\text { Runtime } \\
\text { (in ms) }\end{array}$ & $\begin{array}{l}\text { Memory } \\
\text { (in mb) }\end{array}$ & \\
\hline 0.00167 & 97187 & 11884 & 614.571 & 8002 & 606.597 & Right-to-left \\
\hline 0.00170 & 35174 & 5931 & 326.843 & 4102 & 322.446 & Right-to-left \\
\hline 0.00172 & 9710 & 2263 & 215.494 & 2070 & 191.259 & Right-to-left \\
\hline 0.00200 & 2214 & 1211 & 126.248 & 1401 & 126.246 & Right-to-left \\
\hline 0.00220 & 2146 & 1197 & 126.164 & 1373 & 126.161 & Left-to-right \\
\hline 0.00250 & 2123 & 1168 & 125.961 & 1471 & 125.962 & Left-to-right \\
\hline
\end{tabular}

Table 14: Execution times (in milliseconds) and memory sizes (in mb) performances following the two pattern-growth directions and heuristic prediction on the synthetic dataset data.slen_8.tlen_1.seq.patlen_5.lit.patlen_8.nitems_5000_spmf.

\begin{tabular}{|c|c|c|c|c|c|c|}
\hline \multicolumn{2}{|c|}{ Synthetic dataset } & \multicolumn{2}{|c|}{ Left-to-right } & \multicolumn{2}{|c|}{ Right-to-left } & \multirow{2}{*}{$\begin{array}{l}\text { Heuristic } \\
\text { Prediction }\end{array}$} \\
\hline $\begin{array}{c}\text { Threshold } \\
\text { (in \%) }\end{array}$ & $\begin{array}{l}\text { Pattern } \\
\text { count }\end{array}$ & $\begin{array}{l}\text { Runtime } \\
\text { (in ms) }\end{array}$ & $\begin{array}{l}\text { Memory } \\
\text { (in mb) }\end{array}$ & $\begin{array}{c}\text { Runtime } \\
\text { (in ms) }\end{array}$ & $\begin{array}{l}\text { Memory } \\
\text { (in mb) }\end{array}$ & \\
\hline 0.0010 & 181353 & 18257 & 809.431 & 17556 & 795.277 & Right-to-left \\
\hline 0.0011 & 153240 & 15058 & 754.734 & 13744 & 743.977 & Right-to-left \\
\hline 0.0012 & 88461 & 9623 & 534.108 & 9012 & 539.216 & Right-to-left \\
\hline 0.0013 & 71833 & 7619 & 475.363 & 7299 & 486.217 & Right-to-left \\
\hline 0.0014 & 58953 & 6447 & 423.811 & 6206 & 434.475 & Right-to-left \\
\hline 0.0015 & 48724 & 5666 & 383.937 & 5225 & 393.443 & Right-to-left \\
\hline
\end{tabular}

Table 15: Execution times (in milliseconds) and memory sizes (in mb) performances following the two pattern-growth directions and heuristic prediction on the synthetic dataset data.slen_10.tlen_1.seq.patlen_6.lit.patlen_8.nitems_5000_spmf.

\begin{tabular}{|c|c|c|c|c|c|c|}
\hline \multicolumn{2}{|c|}{ Synthetic dataset } & \multicolumn{2}{|c|}{ Left-to-right } & \multicolumn{2}{|c|}{ Right-to-left } & \multirow{2}{*}{$\begin{array}{c}\text { Heuristic } \\
\text { Prediction }\end{array}$} \\
\hline $\begin{array}{c}\text { Threshold } \\
\text { (in \%) }\end{array}$ & $\begin{array}{c}\text { Pattern } \\
\text { count }\end{array}$ & $\begin{array}{l}\text { Runtime } \\
\text { (in ms) }\end{array}$ & $\begin{array}{l}\text { Memory } \\
\text { (in mb) }\end{array}$ & $\begin{array}{c}\text { Runtime } \\
\text { (in ms) }\end{array}$ & $\begin{array}{c}\text { Memory } \\
\text { (in mb) }\end{array}$ & \\
\hline 0.0014 & 140339 & 10903 & 768.477 & 9851 & 757.935 & Right-to-left \\
\hline 0.0015 & 21536 & 3512 & 293.686 & 3520 & 294.380 & Right-to-left \\
\hline 0.0016 & 9285 & 2497 & 226.348 & 2641 & 224.654 & Right-to-left \\
\hline 0.0017 & 4490 & 2049 & 193.117 & 2222 & 189.378 & Right-to-left \\
\hline 0.0018 & 3284 & 1865 & 178.694 & 2061 & 182.001 & Right-to-left \\
\hline 0.0019 & 2121 & 1704 & 171.187 & 1788 & 171.177 & Left-to-right \\
\hline
\end{tabular}

\subsection{Performance analysis}



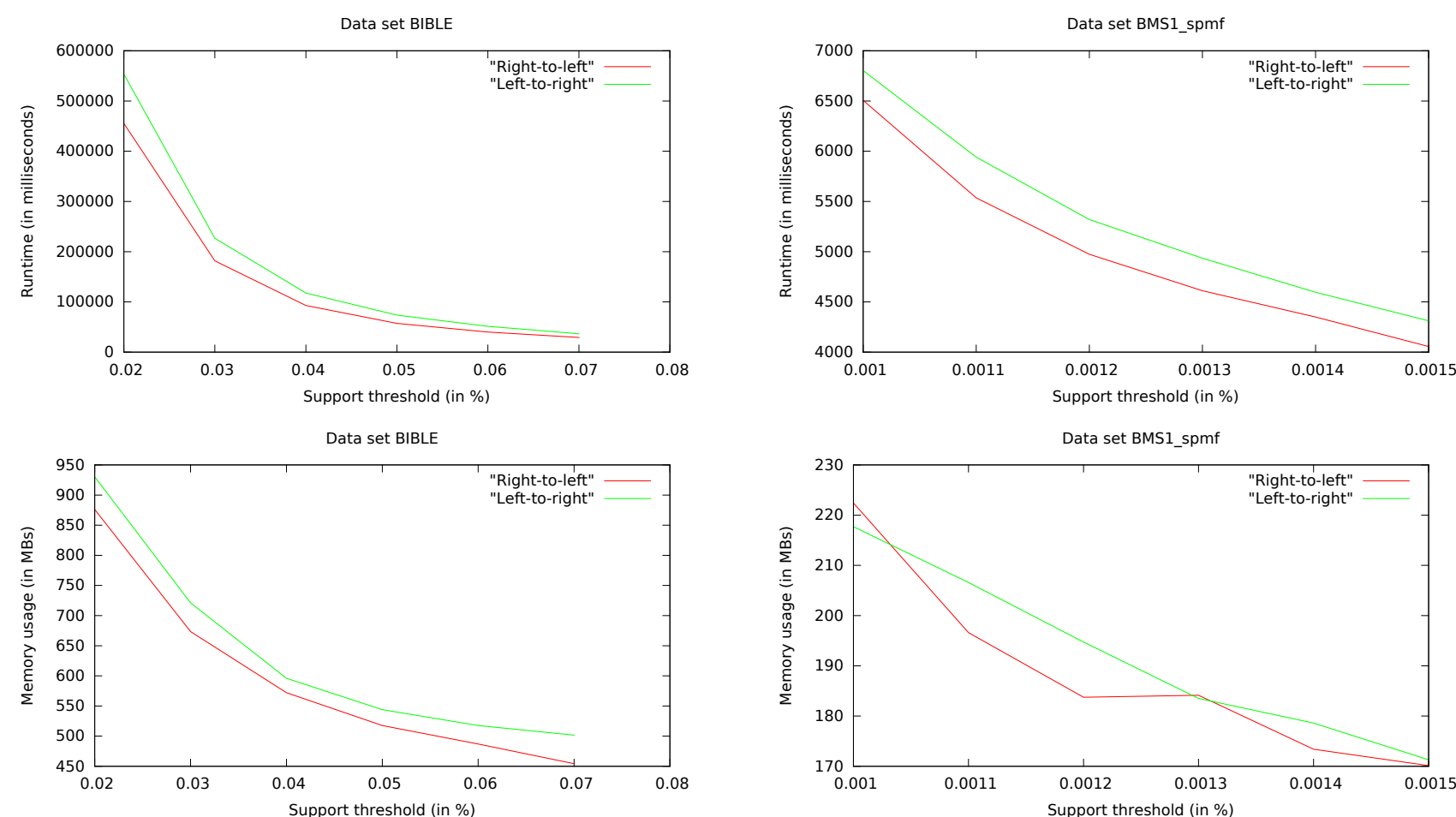

Figure 1: The heuristic recommends the right-to-left pattern-growth direction. It is $1.21-1.25$ times faster and requires almost $1.04-1.10$ times less memory than the other direction.

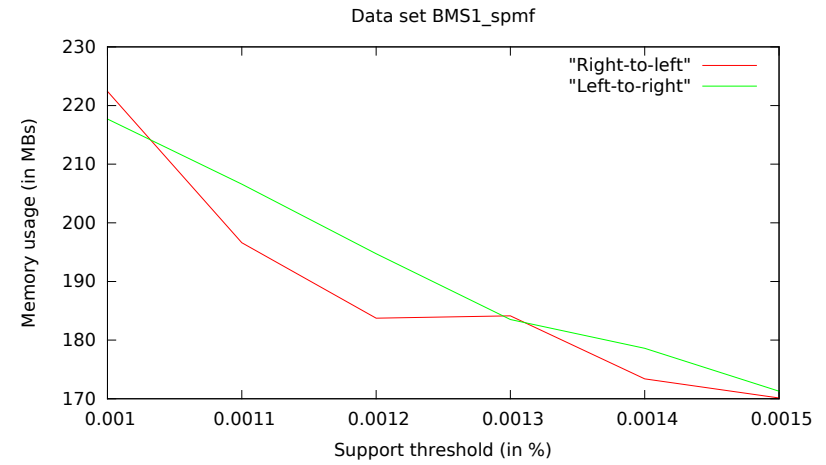

Figure 3: The heuristic recommends the right-to-left pattern-growth direction. It is $1.04-1.05$ times faster. It requires almost $1-1.05$ times less memory than the other direction if the support threshold is greater than 0.001 and a little more memory otherwise.
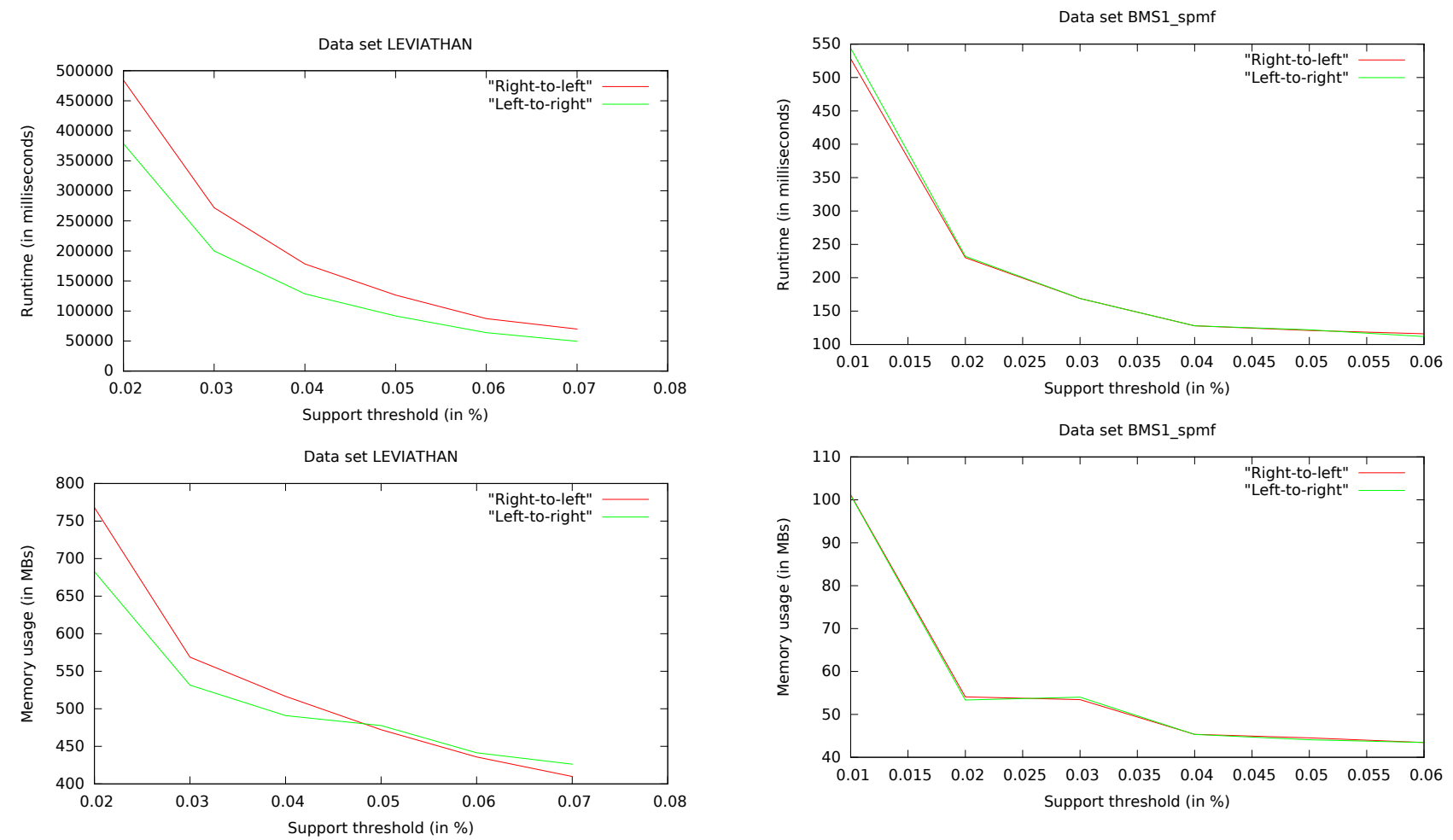

Figure 2: The heuristic recommends the left-to-right pattern-growth direction. It is $1.27-1.4$ times faster, and requires less memory if the support threshold is less than 0.05 and a little more memory otherwise.

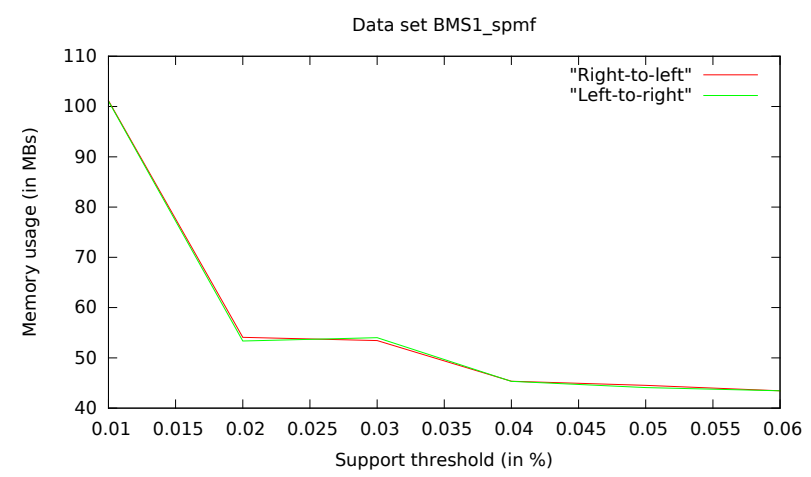

Figure 4: The heuristic recommends the right-to-left pattern-growth direction if the support threshold is within the range $0.01-0.03$ and the left-to-right pattern-growth direction if it is within the range $0.04-0.06$. The runtime (resp. memory usage) performances of the two directions are very close. 

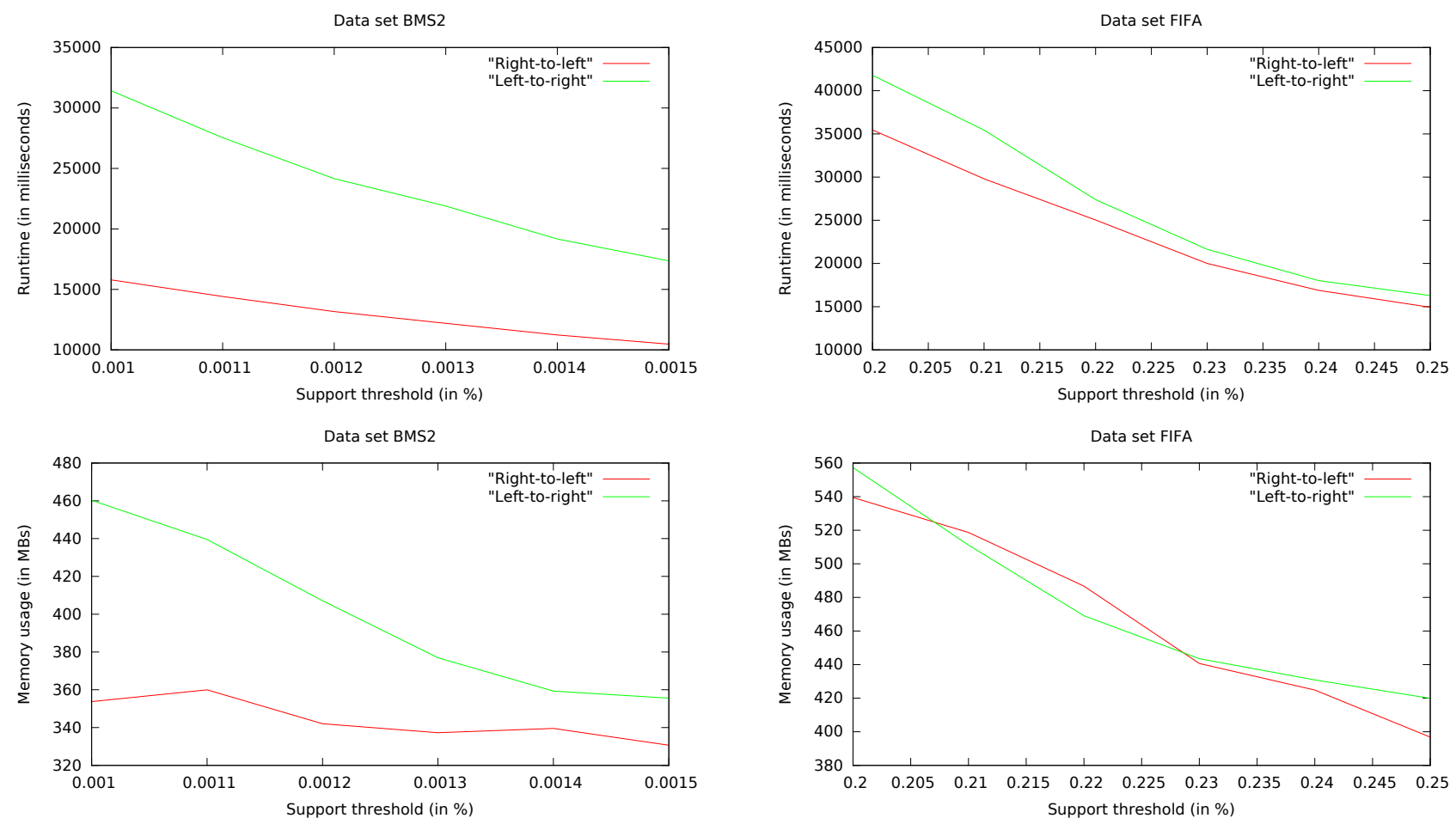

Figure 5: The heuristic recommends the right-to-left pattern-growth direction. It is $1.5-2$ times faster and requires almost $1.07-1.3$ times less memory than the other direction.

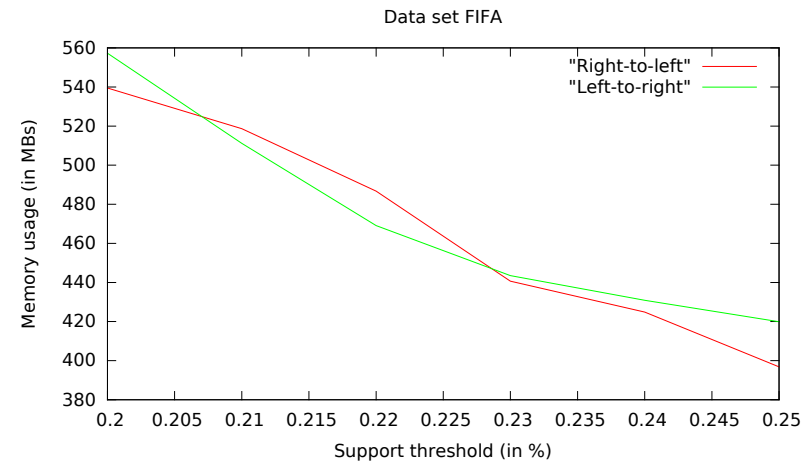

Figure 7: The heuristic recommends the right-to-left pattern-growth direction. It is $1.096-1.178$ times faster. It requires less memory if the support is between $0.206-0.23$ and a little more memory otherwise.
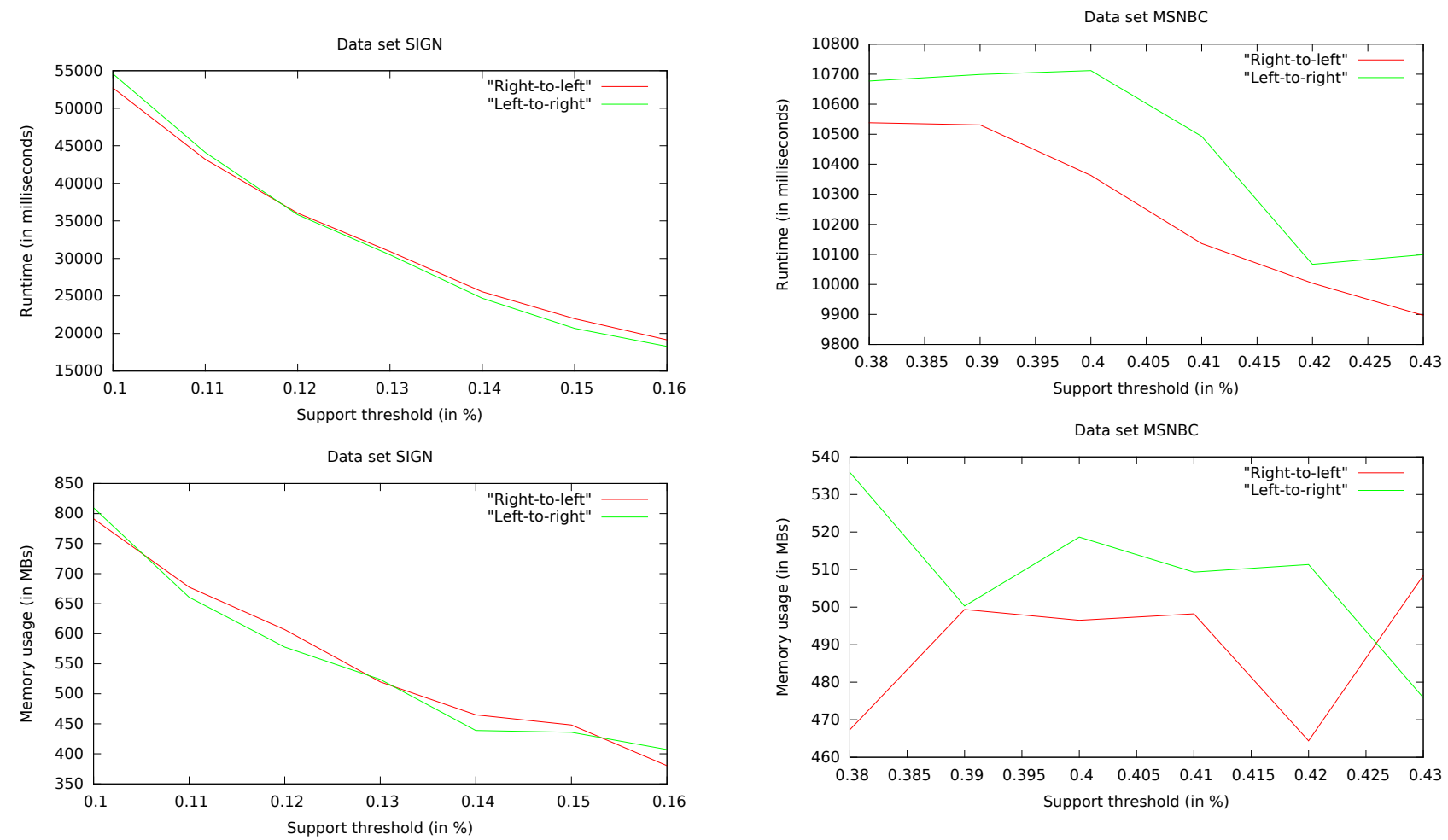

Figure 6: The heuristic recommends the right-to-left pattern-growth direction. It is slightly faster if the support threshold is less than 0.12 and slightly slower otherwise. The memory requirements are close.

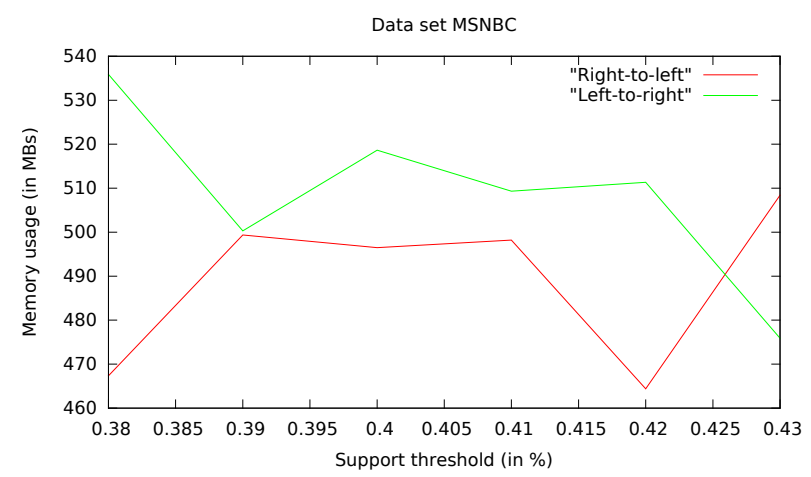

Figure 8: The heuristic recommends the right-to-left pattern-growth direction. It is $1.006-1.033$ times faster and requires almost $1.06-1.14$ times less memory than the other direction. It is slightly slower if the support threshold is more than 0.425 . 

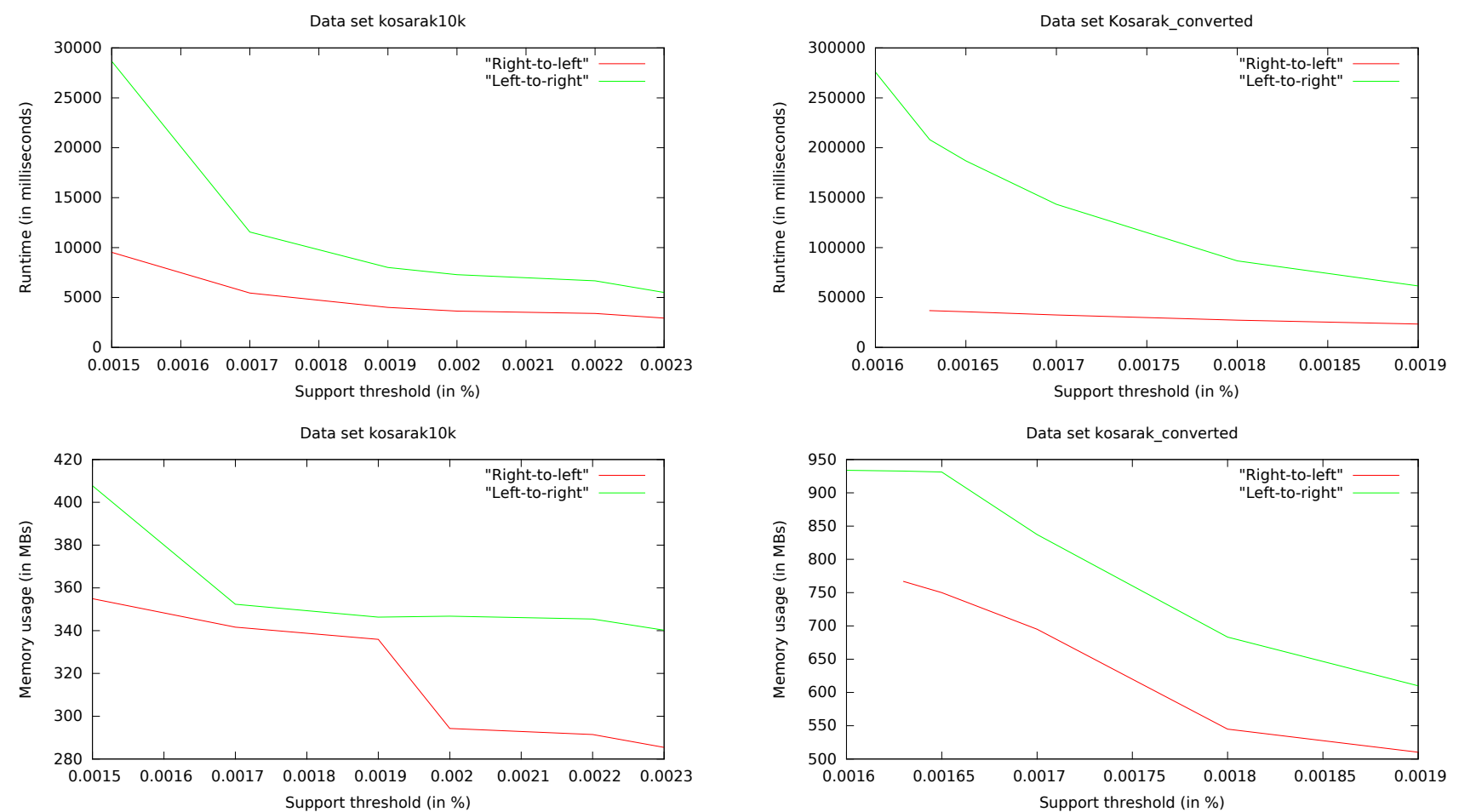

Figure 9: The heuristic recommends the right-to-left pattern-growth direction. It is $1.87-3$ times faster and requires almost $1.03-1.19$ times less memory than the other direction.

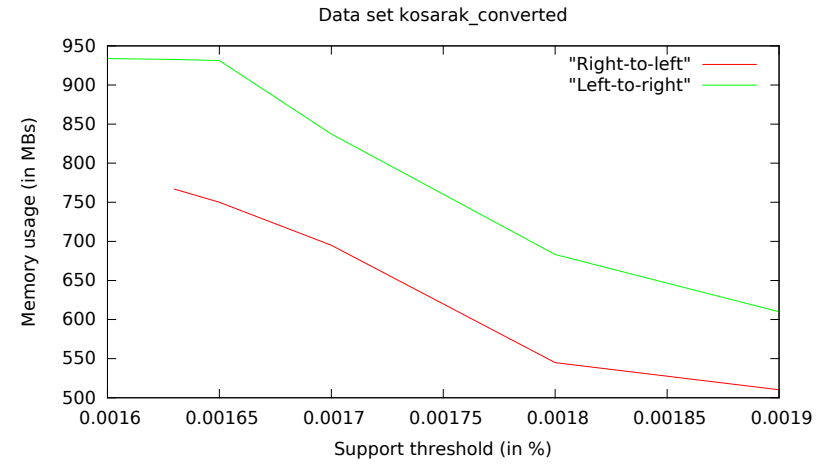

Figure 11: The heuristic recommends the right-to-left direction. It is $2.6-$ 5.6 times faster and requires almost 1.2 times less memory than the other direction.
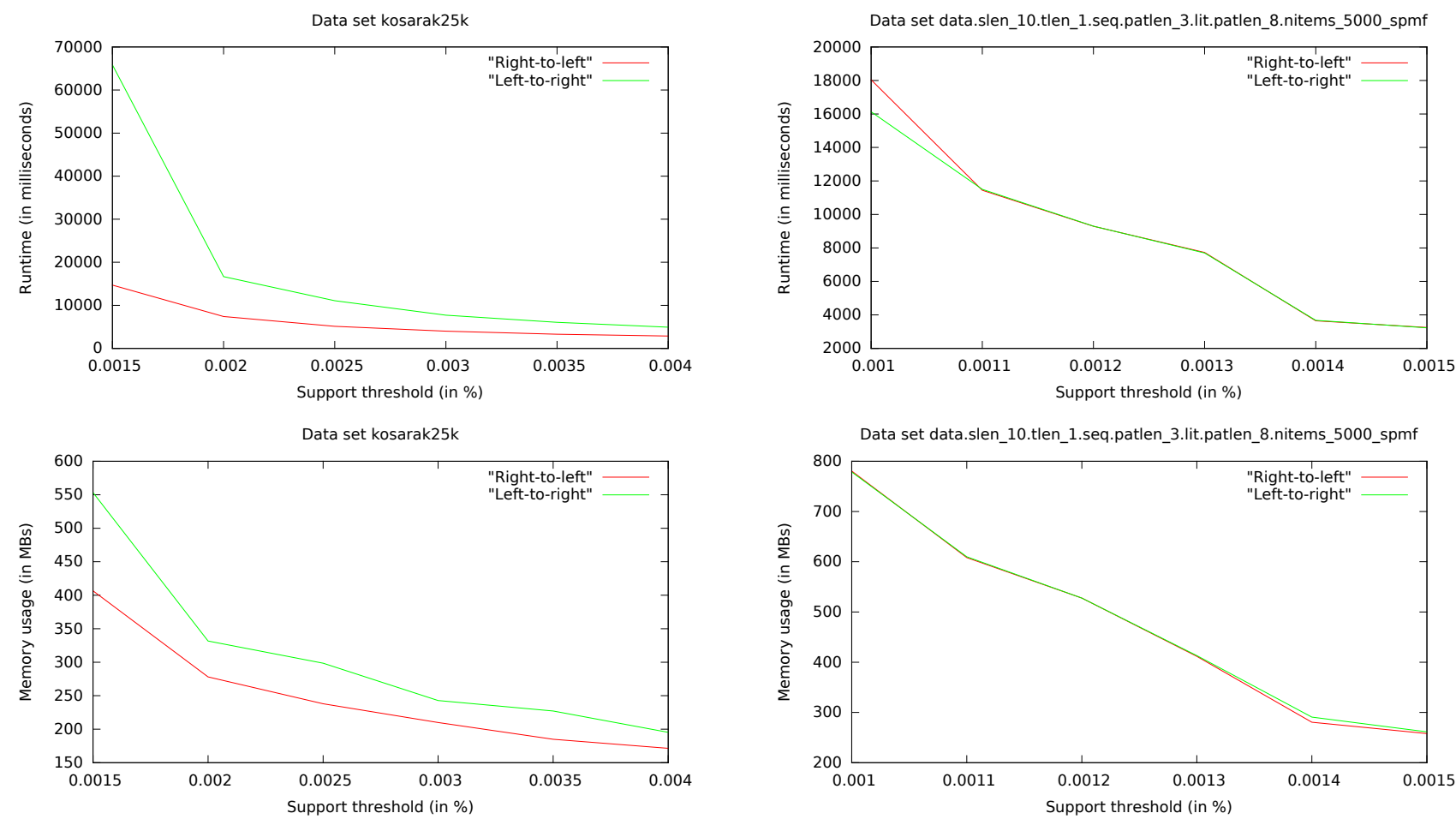

Figure 10: The heuristic recommends the right-to-left direction. It is $1.71-$ 4.47 times faster and requires almost $1.13-1.36$ times less memory than the other direction.

Figure 12: The heuristic recommends the right-to-left direction. It is slightly slower if the support threshold is less than 0.0011. Otherwise the runtimes are very close. The memory requirements are very close. 

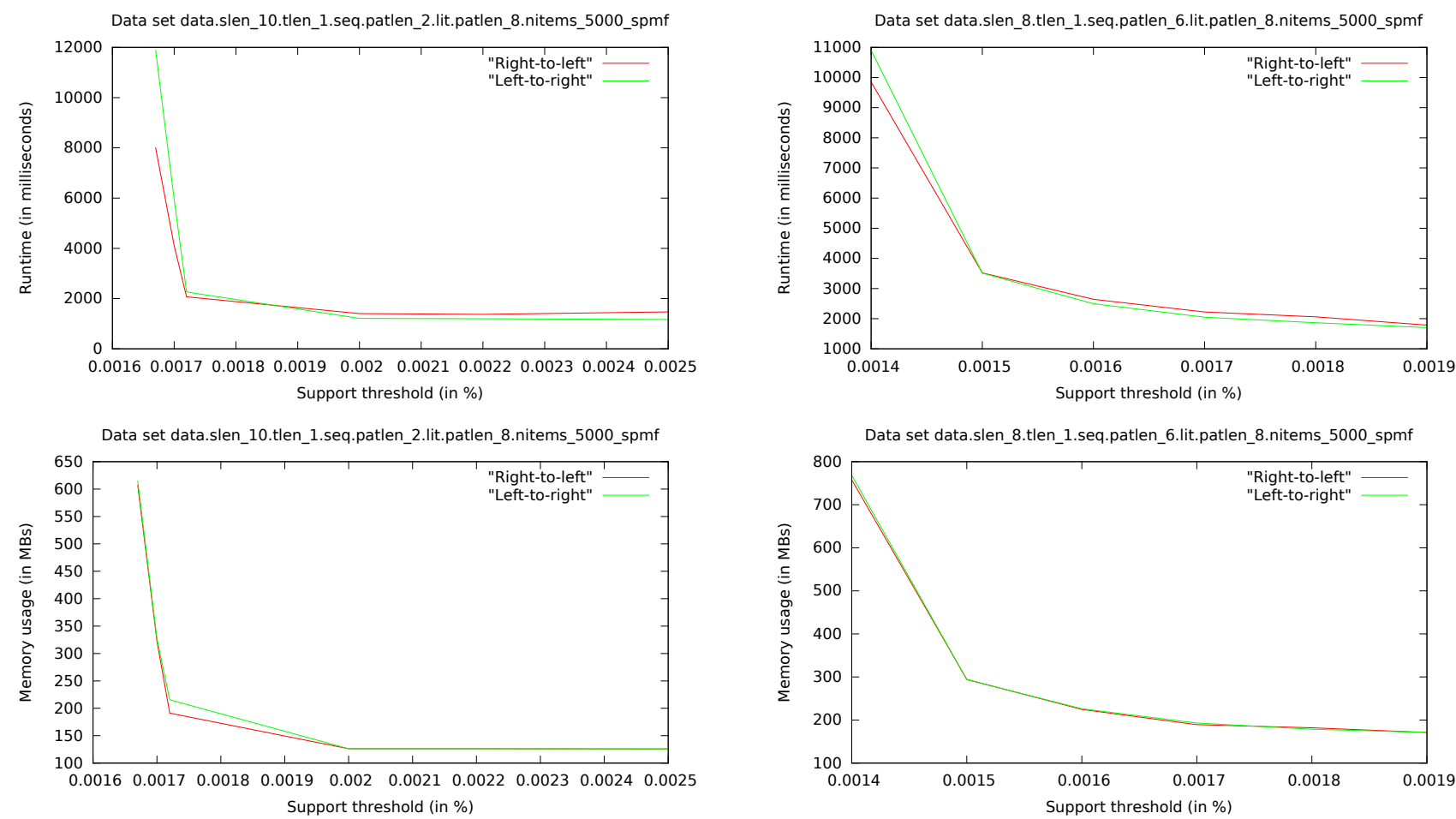

Figure 13: The heuristic recommends the right-to-left direction if the support threshold is within the range $0.00167-0.002$ and the left-to-right direction if it is within the range $0.0022-0.0025$. This prediction is good for three reasons: (1) it requires a little less memory, (2) it is slightly faster if the support threshold is within $0.00167-0.0017$ or $0.0022-0.0025$, and (3) the runtime (resp. memory usage) performances of the two directions are very close.

Data set data.slen 8.tlen_1.seq.patlen_5.lit.patlen_8.nitems_5000_spmf

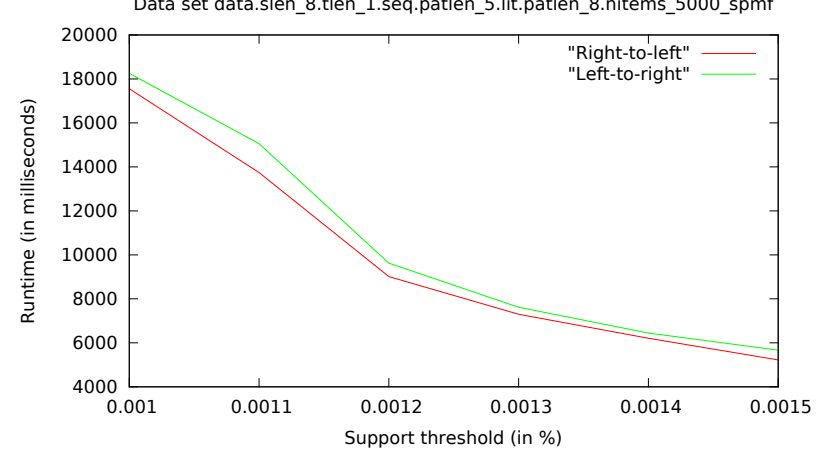

Data set data.slen_8.tlen_1.seq.patlen_5.lit.patlen_8.nitems_5000_spmf

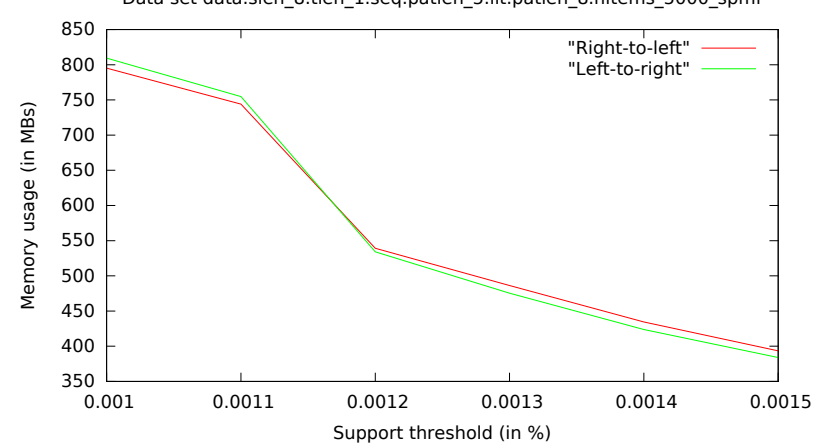

Figure 14: The heuristic recommends the right-to-left pattern-growth direction. It is slightly faster. The memory requirements are very close.

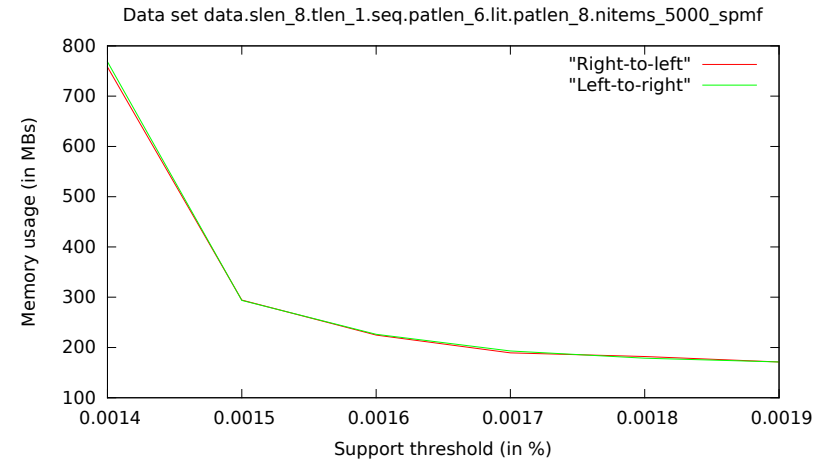

Figure 15: The heuristic recommends the right-to-left direction if the support threshold is within the range $0.0014-0.0018$ and the left-to-right direction if it is within the range $0.0019-0.002$. This prediction is good: (1) it requires almost the same memory, (2) it is slightly faster if the support threshold is less than 0.0015 and (3) the runtime (resp. memory usage) performances of the two directions are very close.

The performance analysis per dataset shows that our heuristic is reliable in general. Thus, it can be used to improve the performances of the pattern growth-based sequential pattern mining approach. The proposed heuristic is based on two tasks: (1) the mining of frequent items and (2) the computation of the cumulative left and right weights of all the sequences in the dataset. Task (1) is included in PrefixSpan $[11,15,16]$ and SuffixSpan $[11,15,16]$ algorithms and task (2) requires only one scan of the dataset. Because of this, the heuristic runtime is negligible compared to the runtine of the two sequential-pattern mining algorithms, i.e PrefixSpan and SuffixSpan.

\section{Conclusion}

In this paper, we have studied the impact of the pattern-growth direction on the performances of the pattern growth-based sequential pattern mining algorithm. Our study show that the pattern-growth direction affect both the runtime and memory usage performances. This has motivated us to introduce a heuristic which recommends a pattern-growth direction between left-to-right direction (also called PrefixSpan) and right-to-left-direction (also called SuffixSpan). Performance analysis related to experimental results obtained from eight real-life datasets and four synthetic datasets, commonly used for evaluating and comparing sequential pattern algorithms, shows that the heuristic prediction is reliable in general. Futhermore the heuristic runtime is negligible compared to the runtine of the two sequential-pattern mining algorithms, i.e PrefixSpan and SuffixSpan.

\section{References}

[1] R. Agrawal, T. Imielinski, and A. N. Swami. Mining association rules between sets of items in large databases. pages 207-216, 1993.

[2] R. Agrawal and R. Srikant. Fast algorithms for mining association rules in large databases. In VLDB'94, Proceedings of 20th International Conference on Very Large Data Bases, September 12-15, 1994, Santiago de Chile, Chile, pages 487-499, 1994. 
[3] R. Agrawal and R. Srikant. Mining sequential patterns. In Proceedings of the Eleventh International Conference on Data Engineering, March 6-10, 1995, Taipei, Taiwan, pages 3-14, 1995.

[4] J. Ayres, J. Flannick, J. Gehrke, and T. Yiu. Sequential pattern mining using a bitmap representation. In Proceedings of the Eighth ACM SIGKDD International Conference on Knowledge Discovery and Data Mining, July 23-26, 2002, Edmonton, Alberta, Canada, pages 429-435, 2002.

[5] T. Dam, K. Li, P. Fournier-Viger, and Q. Duong. An efficient algorithm for mining top-rank-k frequent patterns. Appl. Intell., 45(1):96-111, 2016

[6] M. El-Sayed, C. Ruiz, and E. A. Rundensteiner. Fs-miner: efficient and incremental mining of frequent sequence patterns in web logs. In Sixth ACM CIKM International Workshop on Web Information and Data Management (WIDM 2004), Washington, DC, USA, November 12-13, 2004, pages 128-135, 2004.

[7] P. Fournier-Viger, A. Gomariz, T. Gueniche, A. Soltani, C. Wu, and V. S. Tseng. SPMF: a java open-source pattern mining library. Journal of Machine Learning Research, 15(1):3389-3393, 2014.

[8] M. N. Garofalakis, R. Rastogi, and K. Shim. SPIRIT: sequential pattern mining with regular expression constraints. In VLDB'99, Proceeding of 25th International Conference on Very Large Data Bases, September 7-10, 1999, Edinburgh, Scotland, UK, pages 223-234, 1999.

[9] K. Gouda, M. Hassaan, and M. J. Zaki. Prism: A primal-encoding approach for frequent sequence mining. In Proceedings of the 7th IEEE International Conference on Data Mining (ICDM 2007), October 28 31, 2007, Omaha, Nebraska, USA, pages 487-492, 2007.

[10] K. Gouda, M. Hassaan, and M. J. Zaki. Prism: An effective approach for frequent sequence mining via prime-block encoding. J. Comput. Syst. Sci., 76(1):88-102, 2010.

[11] J. Han, J. Pei, B. Mortazavi-Asl, Q. Chen, U. Dayal, and M. Hsu. Freespan: frequent pattern-projected sequential pattern mining. In Proceedings of the sixth ACM SIGKDD international conference on Knowledge discovery and data mining, Boston, MA, USA, August 20-23, 2000, pages 355-359, 2000.

[12] C. Hsieh, D. Yang, and J. Wu. An efficient sequential pattern mining algorithm based on the 2-sequence matrix. In Workshops Proceedings of the 8th IEEE International Conference on Data Mining (ICDM 2008), December 15-19, 2008, Pisa, Italy, pages 583-591, 2008.

[13] N. R. Mabroukeh and C. I. Ezeife. A taxonomy of sequential pattern mining algorithms. ACM Comput. Surv., 43(1):3, 2010.

[14] F. Masseglia, F. Cathala, and P. Poncelet. The PSP approach for mining sequential patterns. In Principles of Data Mining and Knowledge Discovery, Second European Symposium, PKDD '98, Nantes, France. September 23-26, 1998, Proceedings, pages 176-184, 1998.

[15] J. Pei, J. Han, B. Mortazavi-Asl, H. Pinto, Q. Chen, U. Dayal, and M. Hsu. Prefixspan: Mining sequential patterns by prefix-projected growth. In Proceedings of the 17th International Conference on Data Engineering, April 2-6, 2001, Heidelberg, Germany, pages 215-224, 2001

[16] J. Pei, J. Han, B. Mortazavi-Asl, J. Wang, H. Pinto, Q. Chen, U. Dayal, and M. Hsu. Mining sequential patterns by pattern-growth: The prefixspan approach. IEEE Trans. Knowl. Data Eng., 16(11):1424-1440,

[17] J. Pei, J. Han, B. Mortazavi-Asl, and H. Zhu. Mining access patterns efficiently from web logs. In Knowledge Discovery and Data Mining, Current Issues and New Applications, 4th Pacific-Asia Conference, PADKK 2000, Kyoto, Japan, April 18-20, 2000, Proceedings, pages 396-407, 2000

[18] L. Savary and K. Zeitouni. Indexed bit map (IBM) for mining frequent sequences. In Knowledge Discovery in Databases: PKDD 2005, 9th European Conference on Principles and Practice of Knowledge Discovery in Databases, Porto, Portugal, October 3-7, 2005, Proceedings, pages 659-666, 2005.

[19] M. Seno and G. Karypis. Slpminer: An algorithm for finding frequent sequential patterns using length-decreasing support constraint. In Proceedings of the 2002 IEEE International Conference on Data Mining (ICDM 2002), 9-12 December 2002, Maebashi City, Japan, pages $418-425,2002$.

[20] Z. Yang and M. Kitsuregawa. LAPIN-SPAM: an improved algorithm for mining sequential pattern. In Proceedings of the 21st International Conference on Data Engineering Workshops, ICDE 2005, 5-8 April 2005, Tokyo, Japan, page 1222, 2005.

[21] Z. Yang, Y. Wang, and M. Kitsuregawa. LAPIN: effective sequential pattern mining algorithms by last position induction for dense databases. In Advances in Databases: Concepts, Systems and Applications, 12th International Conference on Database Systems for Advanced Applications, DASFAA 2007, Bangkok, Thailand, April 9-12, 2007, Proceedings, pages 1020-1023, 2007.

[22] M. J. Zaki. Sequence mining in categorical domains: Incorporating constraints. In Proceedings of the 2000 ACM CIKM International Conference on Information and Knowledge Management, McLean, VA, USA, November 6-11, 2000, pages 422-429, 2000.

[23] M. J. Zaki. SPADE: an efficient algorithm for mining frequent sequences. Machine Learning, 42(1/2):31-60, 2001. 